\title{
Attentional capture by motion onsets is modulated by perceptual load
}

\author{
Joshua D. Cosman and Shaun P. Vecera \\ University of Iowa, Iowa City, Iowa
}

\begin{abstract}
The onset of motion captures attention during visual search even if the motion is not task relevant, which suggests that motion onsets capture attention in a stimulus-driven manner. However, we have recently shown that stimulus-driven attentional capture by abruptly appearing objects is attenuated under conditions of high perceptual load. In the present study, we examined the influence of perceptual load on attentional capture by another type of dynamic stimulus: the onset of motion. Participants searched for a target letter through briefly presented low- and high-load displays. On each trial, two irrelevant flankers also appeared, one with a motion onset and one that was static. Flankers defined by a motion onset captured attention in the low-load but not in the high-load displays. This modulation of capture in high-load displays was not the result of overall lengthening of reaction times (RTs) in this condition, since search for a single low-contrast target lengthened RTs but did not influence capture. These results, together with those of previous studies, suggest that perceptual load can modulate attentional capture by dynamic stimuli.
\end{abstract}

In any visual scene, there is more information present than the visual system can process at one time. As a result, specialized attentional mechanisms have evolved that allow us to select and process only the most relevant information in a scene. For example, attention can be selectively focused on the computer screen during typing, while extraneous information in the surrounding environment is ignored. This voluntary-or goal-directed-form of attentional control is central to our ability to carry out goals effectively in complex visual environments. At the same time, it is also possible for salient aspects of stimuli outside of our current focus to capture our attention, redirecting the focus of attention in a stimulus-driven manner. Although stimulus factors play a role in both goaldirected and stimulus-driven attentional control, some have argued that stimulus-driven control is automatic and occurs independent of the goals or beliefs of an observer (e.g., Theeuwes, 1994), whereas others have shown that attentional capture is under the top-down control of the observer (Folk, Remington, \& Johnston, 1992). Thus, it is not clear under what conditions, if any, capture can be purely stimulus driven.

In a number of recent studies, the onset of motion has been demonstrated to capture attention in a stimulusdriven manner, regardless of the observer's goals (Abrams \& Christ, 2003, 2005, 2006; Franconeri \& Simons, 2003, 2005). For example, Franconeri and Simons (2003) showed that a variety of dynamic stimuli could capture attention in a stimulus-driven manner, using an irrelevantfeature search task (Jonides \& Yantis, 1988). Observers initially viewed a five-item placeholder array, and directly following this, portions of each placeholder were removed to reveal a five-item search array. Prior to the presentation of the search array, one of the items in the placeholder array moved, generating a motion onset. Importantly, this motion onset was equally likely to occur at the location of the target or at one of the distractor locations. Across a number of different types of motion onset (e.g., jitter and looming), search slopes to find a target were shallower when the motion onset occurred at the target location than when it occurred at one of the distractor locations. Because this effect was observed despite the task irrelevance of the motion onset, it was concluded that motion onset captures attention in a purely stimulus-driven manner (Franconeri \& Simons, 2003).

In contrast to these results, we have recently demonstrated that salient, task-irrelevant stimuli can be ignored in search arrays that are high in perceptual load (Cosman \& Vecera, 2009). Using displays in which we manipulated perceptual load (cf. Lavie, 1995; Lavie \& Cox, 1997), we showed that abruptly appearing, task-irrelevant flanker letters affected performance in low-load but not in highload displays. This suggests that the attentional processes tapped in high-load displays are needed in order for the effects of capture to be observed.

In the present set of experiments, we seek to generalize our earlier results by asking whether perceptual load affects attentional capture by another class of dynamic stimuli: motion onsets. More specifically, as the difficulty of the search task is increased (i.e., as perceptual load is increased), is there a decrease in the ability of a taskirrelevant motion onset to capture attention? Load theory

J. D. Cosman, joshua-cosman@uiowa.edu 
(Lavie, 1995) would predict that in cases in which attentional demands are high, any task-irrelevant information can be effectively ignored, including dynamic, attentioncapturing stimuli such as motion onsets. Loosely consistent with such an interpretation, Franconeri and Simons (2003) used displays with set sizes of up to seven items, which may be considered high load, and observed steeper search slopes for motion onset targets (i.e., smaller capture effects) for set sizes of five to seven items than at set sizes of three to five items (see Martin-Emerson \& Kramer, 1997, for a similar effect with abrupt onsets). However, in their task, the target item and each distractor were equally likely to appear as motion onsets, and although search slopes increased for motion onset targets with increasing set size, the absolute reaction time (RT) cost (i.e., distractor onset minus target onset RTs) driven by motion onset distractors actually increased at larger set sizes. Therefore, it is hard to tell exactly what effect increases in set size had on capture in Franconeri and Simons's (2003) study. Furthermore, since the motion onset occurred at the target location on some trials, it is possible that the observers showed capture effects because they were reinforced on some trials - albeit infrequently-by having the target appear as a motion onset.

In order to clarify the role of perceptual load on attentional capture, we used a paradigm similar to that used in our previous work (Cosman \& Vecera, 2009). Specifically, we employed a flanker task in which a congruent or incongruent flanker that was completely irrelevant to the task could appear as a motion onset while varying the perceptual load of the search array. On each trial, observers were presented with a briefly presented central search array that contained either one (low load) or six (high load) letters, all defined as offsets, as is shown in Figure 1. The central array was flanked above and below by task-irrelevant flankers, one of which underwent a motion onset in a manner similar to either the translational motion or looming motion conditions used in Franconeri and Simons (2003). If motion onsets capture attention in a purely stimulus-driven manner, the flanker in the location preceded by a motion onset should capture attention regardless of the perceptual load of the display. However, if attentional capture by motion onsets depends on the perceptual load of the display, we would expect to see capture only in the low-load displays.

\section{EXPERIMENT 1}

\section{Method}

Participants. Twenty University of Iowa undergraduates participated in a single session for course credit. All of the observers had normal or corrected-to-normal vision.

Stimuli and Procedure. A Mac mini computer displayed stimuli on a 17-in. CRT monitor and recorded responses and response latencies. The experiment was controlled using MATLAB and the Psychophysics Toolbox (Brainard, 1997).

The observers sat $75 \mathrm{~cm}$ from the monitor in a dimly lit room. In order to determine the effect of perceptual load on attentional capture by motion onsets, we combined Lavie's (1995) perceptual load

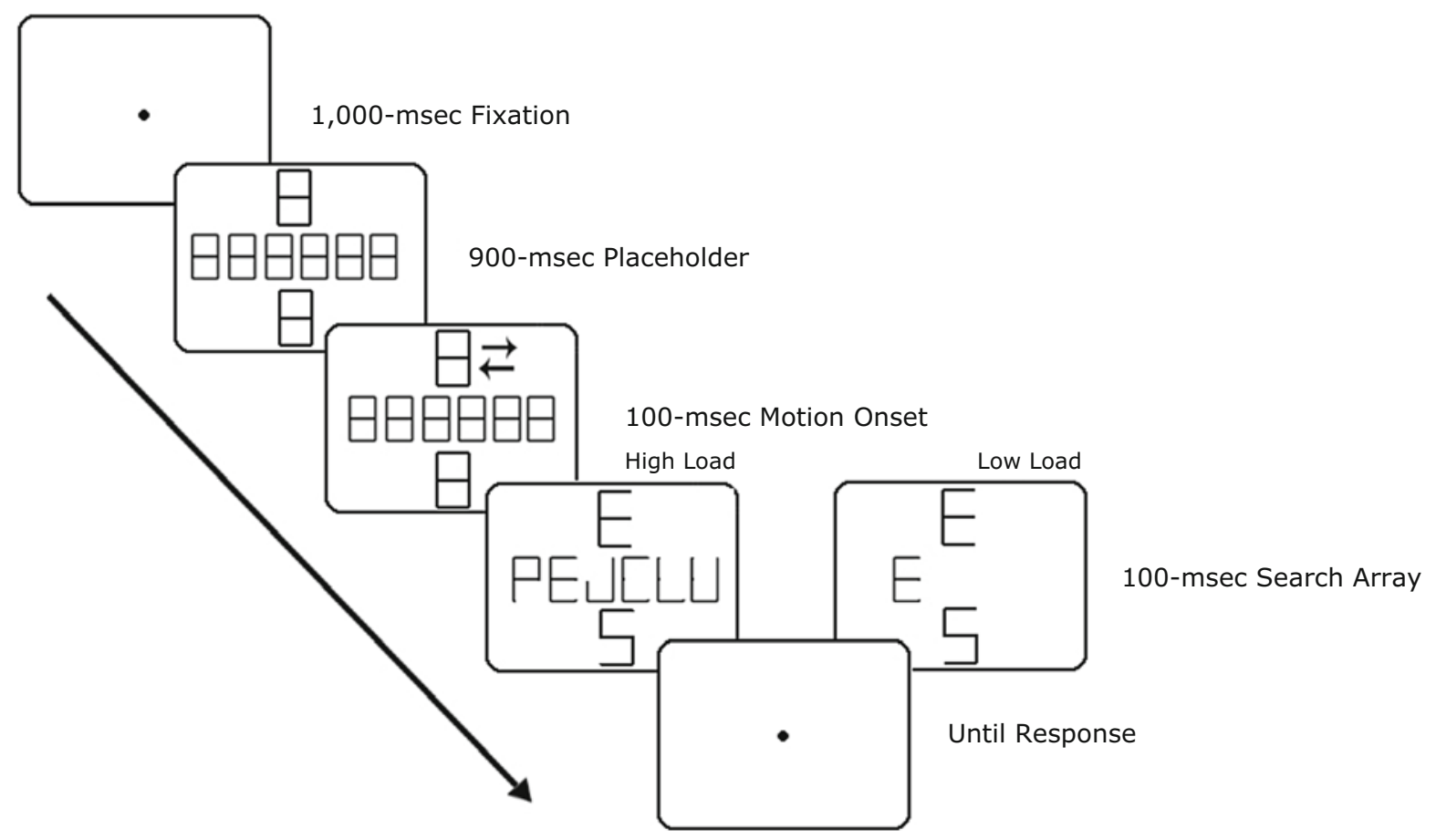

Figure 1. Sequence of events and timing parameters for Experiment 1. Following presentation of a fixation point for 1,000 msec, a six-item placeholder array flanked by two cortically scaled flanker placeholders was presented for 900 msec. Following this, jitter motion occurred at one of the flanker locations for $100 \mathbf{m s e c}$ total, and line segments then disappeared to reveal the search array and flankers, which were presented for $100 \mathrm{msec}$. Search arrays could be high load (six items) or low load (one item). In Experiment 2, timing parameters and stimuli were identical to those in the low-load condition of Experiment 1, but in the lowcontrast condition, the contrast of the target was decreased by $50 \%$ to make the task more difficult. 
paradigm with a motion onset paradigm similar to that used in the jitter motion condition of Experiment 2 of Franconeri and Simons (2003). The procedure and time course of the trials are depicted in Figure 1. At the beginning of each trial, a fixation point measuring $0.35^{\circ} \times 0.35^{\circ}$ appeared for $1,000 \mathrm{msec}$. Next, a placeholder array appeared for $900 \mathrm{msec}$. In both conditions, the placeholder display contained a central array of six figure-eight placeholders subtending $7.50^{\circ} \times 1.60^{\circ}$ of visual angle; each placeholder measured $1.50^{\circ} \times$ $0.75^{\circ}$, with a distance of $0.45^{\circ}$ between placeholders. The placeholders for the flankers, which were scaled according to the cortical magnification factor, measured $1.90^{\circ} \times 0.90^{\circ}$ and appeared above and below the six centrally located placeholders. The center of the flanking placeholder was positioned $3.0^{\circ}$ from the fixation point and $4.5^{\circ}$ from the center of the most eccentric placeholders in the central array.

Directly following the initial presentation of the placeholder array, one of the two flanker placeholders moved to either the left or right by $0.90^{\circ}$ for $50 \mathrm{msec}$ and then returned to its initial position for $50 \mathrm{msec}$. This produced the appearance of a jittering or translational motion, and this motion defined the flanker as having a motion onset. Next, line segments disappeared from the placeholders, including the flanker placeholders, to reveal an array of letters constituting the search array. The resulting high-load display contained two flankers (one of which was accompanied by a motion onset) and six centrally located, task-relevant letters containing a target and five distractors, whereas the low-load display contained two flankers and a single letter (always the target) in one of the six possible locations. This search array remained visible for $100 \mathrm{msec}$, so that the time from the onset of motion to the time of target offset was $200 \mathrm{msec}-$ too brief a duration to permit eye movements.

The observers' task was to report the identity of a target letter, which was either an $\mathrm{E}$ or an $\mathrm{H}$ presented within an array of five distractor letters (U, L, P, C, or J; high-load trials) or by itself (lowload trials). Each letter was equally likely to appear in any of the six different positions in the search array. One of the flanker letters was always the letter $\mathrm{S}$, which was neutral with respect to target (and distractor) identities. The other flanker was either an $\mathrm{E}$ or an $\mathrm{H}$, depending on whether the trial type was congruent or incongruent with the target letter. The observers reported the target's identity by pressing either the " $z$ " or the "/" key, with the response keys corresponding to the $\mathrm{E}$ and $\mathrm{H}$ targets being counterbalanced between observers. Following 48 practice trials, the observers viewed 576 experimental trials. The trials were blocked by load, with each block containing 48 trials of either high- or low-load displays. There were 6 blocks each of high- and low-load displays, resulting in 12 blocks total. The high- and low-load blocks were alternated, and the alternation was counterbalanced such that half of the participants started with high- and the other half started with low-load displays. We informed the observers that the flanker letters were not relevant to the task and stressed that they should be ignored. We also informed the observers to maintain fixation at the center of the screen throughout the experiment.

With this design, it was possible to examine the effects of taskirrelevant motion onset flankers on search performance under conditions of both low and high perceptual load. Specifically, on each trial, the central search array was flanked by letters that appeared both above and below the array. Only one of the placeholders moved prior to the onset of the search array, and this was always defined as the motion onset flanker. The motion onset and static flankers were the neutral letter S ( $50 \%$ of trials) or one of the target letters (E or $\mathrm{H} ; 50 \%$ of trials) and were equally likely to appear above or below the search array. Thus, flanker effects provided a means of assessing capture by motion onsets: To the extent that a flanker effect was observed under these conditions, it could be said that capture occurred.

\section{Results and Discussion}

For each observer, we computed trimmed mean RTs and excluded any RTs under $200 \mathrm{msec}$ or over 3,000 msec. This trimming eliminated less than $2 \%$ of the data. Only correct responses were analyzed. Figure 2 shows mean trimmed RTs and error rates. Both accuracy and RT data were analyzed with a three-factor repeated measures ANOVA, with display load (high vs. low), motion onset

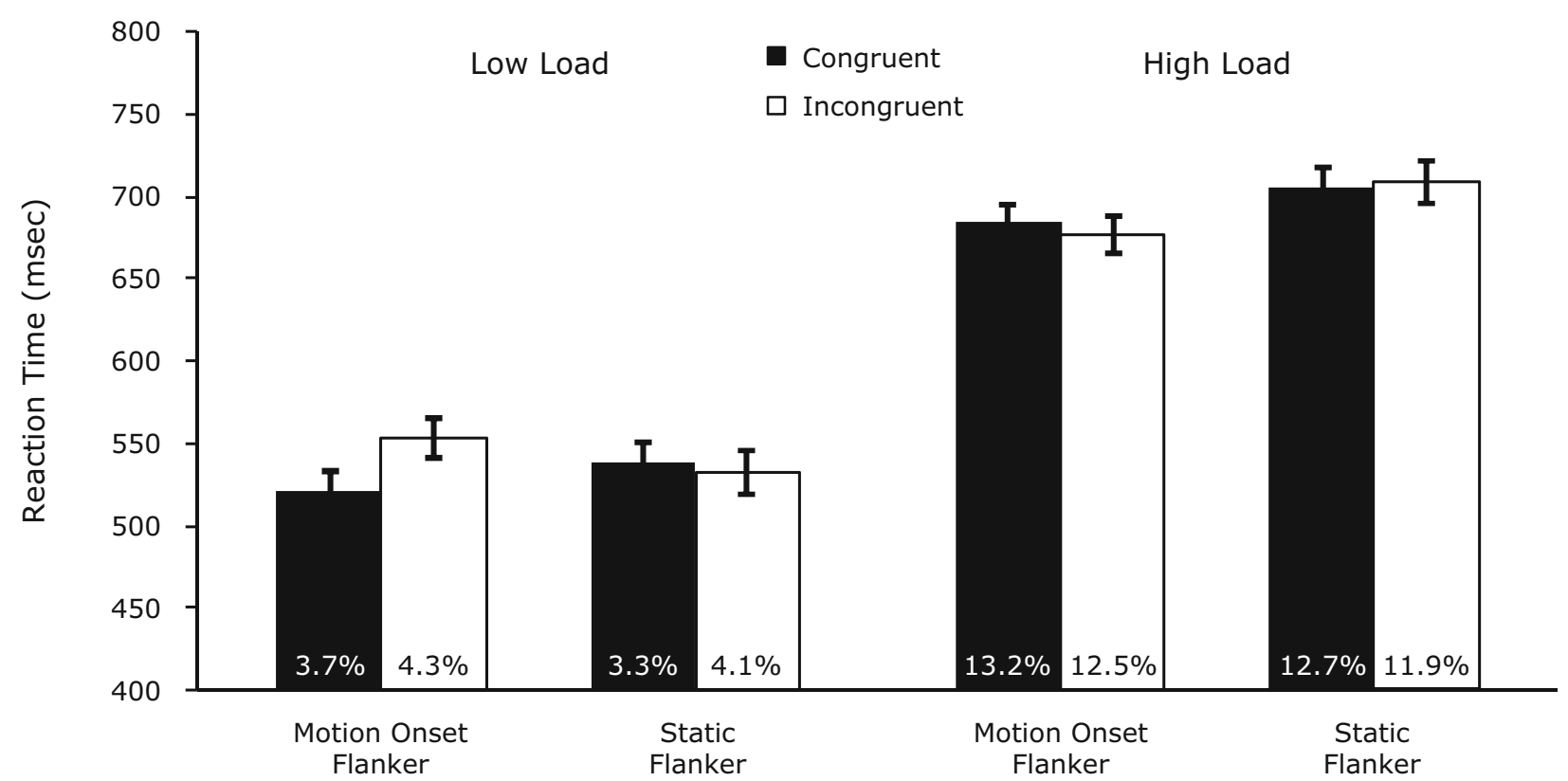

Figure 2. Mean reaction times for high- and low-load conditions when flankers were jitter motion onsets or nononsets in Experiment 1. Error rates for each condition are indicated at the base of the graph. Error bars represent $95 \%$ within-subjects confidence intervals for the flanker effect for each condition (Cousineau, 2005; Loftus \& Masson, 1994). 
status (motion onset vs. static flanker), and flanker congruency (congruent vs. incongruent) as factors.

For RTs, we observed a main effect of load $[F(1,19)=$ $117.1, p<.0001]$, where RTs in the low-load condition were shorter overall $(536 \mathrm{msec})$ than those in the highload condition $(693 \mathrm{msec})$. There were no main effects for motion onset status $[F(1,19)<1$, n.s.] or congruency $[F(1,19)<1$, n.s.], and there were no two-way interactions between load and motion onset status $[F(1,19)<1$, n.s.], load and flanker congruency $[F(1,19)=1.7$, n.s.], or motion onset status and flanker congruency $[F(1,19)<1$, n.s.]. However, the three-way interaction between load, motion onset status, and congruency was significant $[F(1,19)=7.5, p=.01]$, indicating that motion onset capture depended on the level of perceptual load.

A secondary two-way ANOVA with load and congruency as factors was performed on RTs from motion onset trials alone, and the results of this analysis revealed a significant main effect of load $[F(1,19)=30.4, p<.0001]$ but no main effect of congruency $[F(1,19)=2.2$, n.s.] However, the two-way interaction between load and congruency was highly significant $[F(1,19)=10.5, p<.01]$, indicating that within motion onset trials, the magnitude of the flanker effect depended on load. Importantly, as can be seen in Figure 2, attentional capture by an irrelevant distractor was observed only in the low-load motion onset condition, as was confirmed with planned comparisons between congruent and incongruent flanker conditions for each of the low-load motion onset conditions. There was a significant flanker effect in the low-load motion onset condition $[t(19)=3.0, p<.01]$ but not in the high-load motion onset condition $[t(19)<1$, n.s.]. Therefore, it appears that motion onsets only interfered with task performance in the low-load condition.

For error rates, there was a significant main effect of load, with the participants showing higher error rates in the high-load condition $(11.5 \%)$ than in the low-load condition $(2.7 \%)[F(1,19)=55.6, p<.0001]$. No other main effects or interactions were significant $(F \mathrm{~s}<1.2$, $p$ s $>.30)$.

The results indicate that attention capture by motion onsets was modulated by perceptual load. When the observers searched through the high-load displays, they were not captured by irrelevant stimuli with a motion onset. However, motion onsets captured attention when the observers searched displays in which the target appeared alone and search required relatively few attentional resources. These results are in line with the results of Cosman and Vecera (2009) and indicate that perceptual load can affect attention capture by motion onsets.

Although the results of Experiment 1 show that onset capture can be modulated by perceptual load, there is a possibility that capture by motion onsets in the high-load condition was obscured by the longer RTs in that condition. In particular, the lengthening of RTs in the high-load condition may have masked evidence of capture by motion onsets. To ensure that this was not the case, in Experiment 2 , we manipulated task difficulty without varying perceptual load. We created a data-limited situation by using low-contrast target letters that would increase RTs; such a data limitation contrasts with resource-limited situations, in which the resources of some cognitive operation (e.g., attention) are limited (Lavie \& de Fockert, 2003; Norman \& Bobrow, 1975).

Specifically, in Experiment 2, observers viewed arrays that were identical to those in the low-load condition of Experiment 1, but in half of the blocks, the luminance contrast of the single target letter was decreased in order to increase perceptual demands and to make the task more difficult without increasing the capacity load of the displays. Such a manipulation has been used previously in the context of perceptual load (Lavie \& de Fockert, 2003) in order to dissociate the effects of general perceptual discrimination difficulty and the capacity-limited attention mechanisms tapped by perceptual load.

If the results of Experiment 1 are due solely to longer RTs resulting from the more difficult high-load search task, we would expect to see evidence of capture by motion onsets in only the easier high-contrast condition. Alternatively, if our results truly reflect a capacity-dependent modulation of capture by motion onsets, we would expect to see evidence of capture in both the high- and low-contrast conditions, since both include only a single briefly presented search item and are low in perceptual load.

\section{EXPERIMENT 2}

\section{Method}

Participants. Twenty University of Iowa undergraduates participated in a single session for course credit. All of the observers had normal or corrected-to-normal vision.

Stimuli and Procedure. The overall design of Experiment 2 was identical to that of the low-load condition of Experiment 1 . In the high-contrast condition, the trials were identical to those in the lowload condition of Experiment 1. In the low-contrast condition, the luminance contrast of the target letter was decreased by $50 \%$ relative to that in the high-contrast condition to increase task difficulty. Contrast was blocked, just as load was blocked in Experiment 1.

\section{Results and Discussion}

The data were trimmed as in Experiment 1. This trimming eliminated less than $3 \%$ of the data. Only correct responses were analyzed. Figure 3 shows mean trimmed RTs and error rates. Both accuracy and RT data were analyzed as in Experiment 1.

For RTs, we observed a main effect of contrast $[F(1,19)=150.6, p<.0001]$, in which RTs in the highcontrast condition were shorter overall $(524 \mathrm{msec})$ than those in the low-contrast condition $(663 \mathrm{msec})$. There were significant main effects of motion onset status $[F(1,19)=7.7, p=.01]$ and flanker congruency $[F(1,19)=18.2, p=.0001]$ and a significant interaction between motion onset status and flanker congruency $[F(1,19)=7.7, p<.01]$. There was no interaction between contrast and motion onset status $[F(1,19)=1.6$, $p>.20]$ or between contrast and flanker congruency $[F(1,19)=1.7, p>.20]$, nor was there a three-way interaction among contrast, motion onset status, and flanker congruency $[F(1,19)<1$, n.s.].

As in Experiment 1, a secondary two-way ANOVA with load and congruency as factors was performed on 


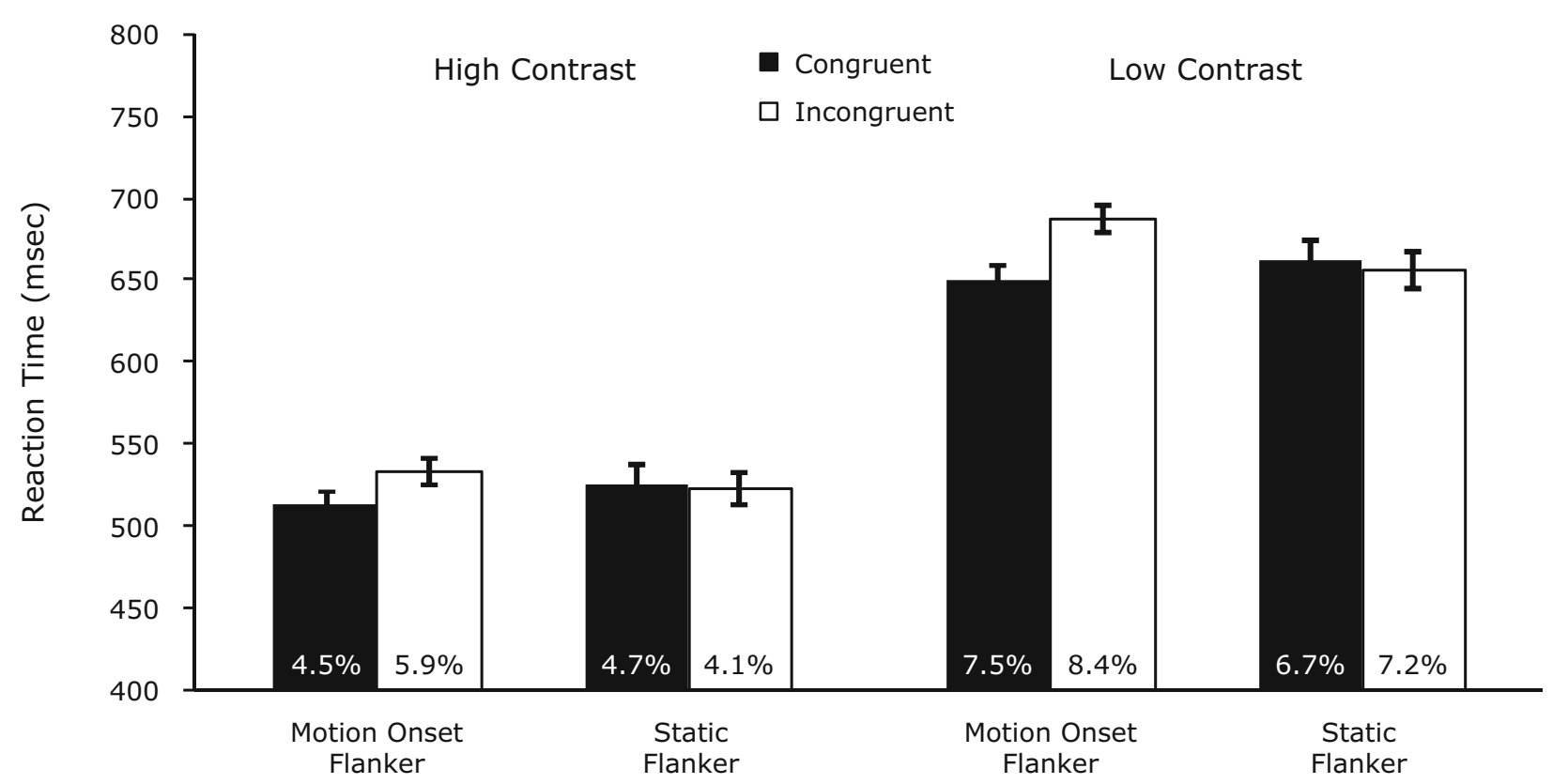

Figure 3. Mean reaction times for high-contrast (easy search) and low-contrast (difficult search) target conditions in Experiment 2. Error rates for each condition are indicated at the base of the graph. Error bars represent $95 \%$ within-subjects confidence intervals for the flanker effect in each condition (Cousineau, 2005; Loftus \& Masson, 1994).

RTs from the motion onset trials alone, and the results of this analysis revealed a significant main effect of contrast $[F(1,19)=334.5, p<.0001]$ and a main effect of congruency $[F(1,19)=12.9, p<.01]$. However, there was no significant interaction between contrast and congruency $[F(1,19)=1.1$, n.s.], indicating that within onset trials, the magnitude of the flanker effect was similar in the low- and high-contrast conditions. As can be seen in Figure 3, attentional capture by an irrelevant distractor was observed in both the easy high-contrast and the more difficult low-contrast conditions, as was confirmed with planned comparisons on the congruent versus incongruent conditions for each of the four motion-onset-by-load conditions. There were significant flanker effects in the low-contrast motion onset condition $[t(19)=2.4, p=.02]$ and in the high-contrast motion onset condition $[t(19)=$ $2.8, p=.01]$ but not in either of the static conditions $(t \mathrm{~s}<$ $1.2, p s>.26)$. Therefore, it appears that the modulation of capture that we observed in Experiment 1 was not due to overall RT lengthening but was instead due to an increase in perceptual load in the high-load displays.

Error rates did not differ significantly between the motion onset and static flanker conditions $[F(1,19)<1$, n.s. $]$ or between the congruent and incongruent flanker conditions $[F(1,19)<1$, n.s.]. However, there was a significant main effect of contrast on error rates, with the participants showing slightly higher error rates in the low-contrast condition $(7.4 \%)$ than in the high-contrast condition $(4.9 \%)$ $[F(1,19)=4.1, p<.05]$. There were no other significant main effects or interactions $(F \mathrm{~s}<3.2, p \mathrm{~s}>.08)$.

The results indicate that attentional capture by motion onsets is modulated by perceptual load. In contrast to Experiment 1, motion onsets captured attention in both the difficult low-contrast condition and the easier highcontrast condition. Therefore, the results of Experiment 1 were not due to an overall lengthening of RTs in the highload condition. These results are in line with the results of Cosman and Vecera (2009) and indicate that perceptual load can affect attention capture by motion onsets. However, although the magnitude of the flanker effect in the low-contrast condition ( $36 \mathrm{msec}$ ) was numerically larger than that in the high-contrast condition $(20 \mathrm{msec})$, this difference was not statistically significant $[t(19)=1.2$, $p=.23]$. This is not consistent with the results of Experiment 2 of Lavie and de Fockert (2003), who used a similar contrast manipulation and obtained larger flanker effects in the degraded low-load (low-contrast) condition than in the normal low-load (high-contrast) condition. However, given the higher overall error rates in Experiment 2 of Lavie and de Fockert's study $(\sim 20 \%)$ relative to those in the present experiment (7.4\%), it is possible that our degradation manipulation was not strong enough to replicate their load effect on accuracy. Regardless, our manipulation was sufficient to increase overall RTs in the degraded low-load condition by $139 \mathrm{msec}$ relative to the normal low-load condition, demonstrating that the lack of a flanker effect in the high-load condition of Experiment 1 was not the result of overall lengthening of RTs.

With the effect of load established, in Experiment 3, we sought to test the generality of our results by examining the effect of load on a different type of motion onset: looming motion. Given that looming motion has been hypothesized to result in capture effects because of its behavioral urgency (Franconeri \& Simons, 2003; but see Abrams \& Christ, 2005), this represents a particularly strong test case for load-dependent capture by motion onsets. 


\section{EXPERIMENT 3}

\section{Method}

Participants. Twenty University of Iowa undergraduates participated in a single session for course credit. All of the observers had normal or corrected-to-normal vision.

Stimuli and Procedure. The overall design of Experiment 3 was identical to that of Experiment 1, with the exception that the motion onset was defined by looming motion. The looming motion was created by having one of the two flankers start at $20 \%$ of the size of the flanker placeholder on the opposite side of the array, with this placeholder gradually growing until it was the same size as the other flanker placeholder. After a 1,000-msec fixation display, a placeholder array similar to that in Experiment 1 appeared for $900 \mathrm{msec}$ with one of the two flanker placeholders presented at $20 \%$ of the size of the other. Over the course of the ensuing $100 \mathrm{msec}$, the motion onset flanker placeholder increased in size in 20 -msec frames until it was equal in size to the other placeholder, giving the impression that this placeholder was moving toward the observer. All other aspects of Experiment 3 were identical to those of Experiment 1.

\section{Results and Discussion}

The data were trimmed as in Experiments 1 and 2. This trimming eliminated less than $3 \%$ of the data. Only correct responses were analyzed. Figure 4 shows mean trimmed RTs and error rates. Both accuracy and RT data were analyzed as in Experiments 1 and 2.

Overall, the results parallel those from Experiment 1 and suggest that looming motion, like translational motion, only captures attention under low-load conditions. For RTs, we observed a main effect of load $[F(1,19)=$ $133.8, p<.0001$ ], where RTs in the low-load condition were shorter overall $(501 \mathrm{msec})$ than those in the highload condition $(634 \mathrm{msec})$. There were no main effects for motion onset status $[F(1,19)<1$, n.s.] or congruency $[F(1,19)<1$, n.s. $]$, but the two-way interactions between load and motion onset status $[F(1,19)<8.0, p<.05]$, load and flanker congruency $[F(1,19)=12.2, p<.01]$, and motion onset status and flanker congruency $[F(1,19)=$ 22.0, $p<.001]$ were all significant. Importantly, the three-way interaction between load, motion onset status, and congruency was significant $[F(1,19)=5.2, p<.05]$, indicating that flanker effects driven by motion onset capture depended on both the level of perceptual load and the flanker's status as a motion onset. A secondary two-way ANOVA with load and congruency as factors was performed on RTs from motion onset trials alone, and the results of this analysis revealed a significant main effect of load $[F(1,19)=98.4, p<.0001]$ and a main effect of congruency $[F(1,19)=26.8, p<.0001]$. In addition, the twoway interaction between load and congruency was highly significant $[F(1,19)=20.6, p<.001]$, indicating that within motion onset trials, the magnitude of the flanker effect depended on load. Importantly, as can be seen in Figure 4, attentional capture by an irrelevant distractor was observed only in the low-load motion onset condition, which was confirmed with planned comparisons on the congruent versus incongruent conditions for each of the low-load motion onset status conditions. There was a significant flanker effect only in the low-load motion onset condition $[t(19)=9.5, p<.0001]$, not in the highload motion onset condition $[t(19)<1$, n.s.], indicating that flankers defined by motion onset interfered with task performance only in the low-load condition.

Error rates did not differ significantly between the congruent and incongruent flanker conditions $[F(1,19)<1$, n.s.], and none of the interactions was significant $\left(F_{\mathrm{S}}<\right.$ $2.4, p s>.13)$. There was a significant main effect of load on error rates, with the participants showing higher error rates in the high-load condition $(11.5 \%)$ than in the low-

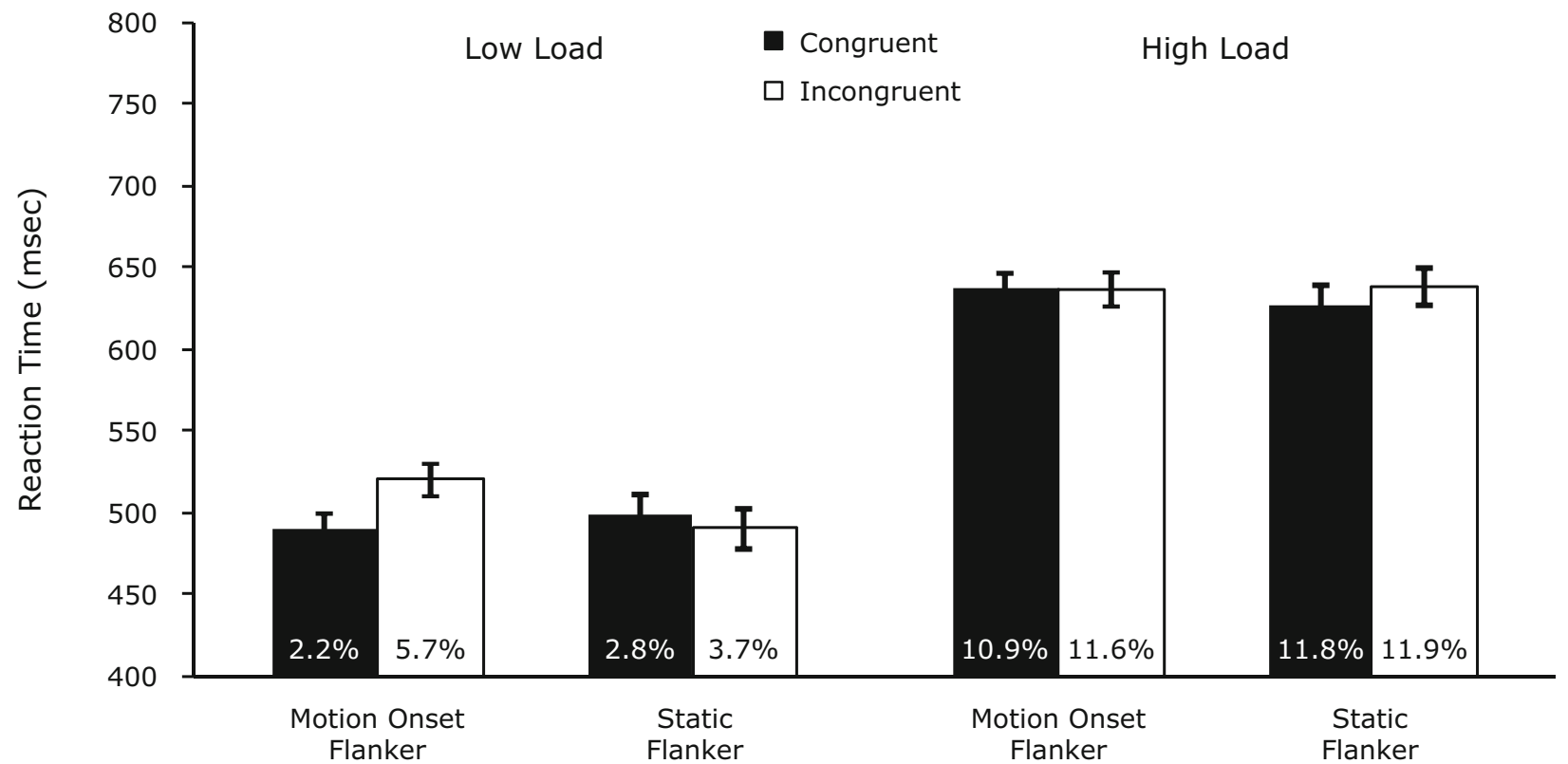

Figure 4. Mean reaction times for high- and low-load conditions when flankers were looming motion onsets or nononsets in Experiment 3. Error rates for each condition are indicated at the base of the graph. Error bars represent $95 \%$ within-subjects confidence intervals for the flanker effect for each condition (Cousineau, 2005; Loftus \& Masson, 1994). 
load condition $(2.7 \%)[F(1,19)=29.2, p<.0001]$. In addition, there was a significant effect of flanker congruency on error rates $[F(1,19)=7.1, p<.01]$, most likely driven by the highly significant difference in accuracy across congruency conditions in the low-load motion onset condition $[t(19)=3.8, p=.001]$; none of the other planned comparisons performed on the accuracy data was significant $(t \mathrm{~s}<0.83, p \mathrm{~s}>.42)$.

The results indicate that the effects of load on capture by motion onsets generalize across different types of motion. When the observers searched through high-load displays, their attention was not captured by irrelevant stimuli with a motion onset, even when that irrelevant stimulus was defined by looming motion. Although looming motion is a potent signal that can capture attention, such capture appears to emerge only in low-load displays.

\section{GENERAL DISCUSSION}

In the present set of experiments, we demonstrated that perceptual load modulates the ability of motion onsets to capture attention. When participants perform an attentionally demanding search task high in perceptual load, motion onsets fail to produce a flanker effect, although the ability of motion onsets to drive a flanker effect is preserved when the participants perform a less demanding, low-perceptual-load search. This represents the first demonstration that attention capture by the onset of new motion is not purely stimulus-driven and complements other results showing a similar interaction between perceptual load and capture by abrupt onsets (Cosman \& Vecera, 2009; Santangelo, Finoia, Raffone, Belardinelli, \& Spence, 2008; Santangelo \& Spence, 2007) and by color singletons (Gibson \& Bryant, 2008; but see Biggs \& Gibson, 2010).

\section{Relationship With Previous Studies of Perceptual Load}

In contrast to traditional demonstrations of control over capture, the effects of load on capture represent a special case, in that they are not top-down in the traditional sense. Because perceptual load effects are typically described in terms of the limited-capacity mechanisms of perceptual-level attention, our results differ from those of previous demonstrations of control over capture (e.g., an attentional set; Folk et al.,1992; Leber \& Egeth, 2006), in that the locus of the effect is hypothesized to be perceptual in nature (Lavie, Hirst, de Fockert, \& Viding, 2004). As a result, the load effects that we have observed here and in our previous work (Cosman \& Vecera, 2009) may be conceptualized as leading to a bottom-up - rather than a top-down - attenuation of capture: To the extent that there are more task-relevant items than can be perceptually processed during search, there will be no room for the perceptual processing of task-irrelevant items, despite their salience. Such a description of load effects would suggest that the attenuation of capture during high-load search is driven by early, bottom-up perceptual factors. In support of this interpretation, recent results show that low-level stimulus factors, such as crowding, drive perceptual load effects (see, e.g., Torralbo \& Beck, 2008), with increased crowding producing higher perceptual load. Thus, the attenuation of attentional capture under high-load conditions may be a bottom-up effect.

However, a more complete view would be that the effects of load on attentional capture may be better conceptualized as arising from interactions between top-down and bottom-up processes. Recent evidence from our lab (Cosman \& Vecera, in press) and others (Biggs \& Gibson, 2010; Couperus, 2009; Theeuwes, Kramer, \& Belopolsky, 2004) demonstrates that load effects are at least in part driven by top-down attentional control settings. For example, Theeuwes et al. showed that intertrial expectancies play a role in perceptual load effects. In their study, Theeuwes et al. intermixed high- and low-load displays to examine possible carryover effects between low- and high-load trials. Critically, attenuated flanker effects were only observed when a high-load trial was preceded by another high-load trial; when a high-load trial was preceded by a low-load trial, a flanker effect was observed, despite the fact that the current display had a high perceptual load. This indicates that top-down control settings driven by short-term expectancies play a role in establishing perceptual load. Along these same lines, we have recently shown that the frequency with which a task-irrelevant flanker appears as an onset affects whether it will capture attention and produce a flanker effect under conditions of high load (Cosman \& Vecera, in press). Specifically, if a task-irrelevant flanker appears as an onset on $80 \%$ of trials, the onsets fail to capture attention; however, if the flanker is presented as an onset on only $20 \%$ of trials, the onsets capture attention strongly, interfering with task performance. Therefore, top-down expectancies regarding the search task are at least partially responsible for typical high-load effects, with such effects reflecting contributions from both bottom-up and top-down processes.

Regardless of the exact mechanisms responsible for driving load effects, our data provide at least partial support for load theory (Lavie, 1995; Lavie et al., 2004), in that under conditions of high perceptual load, a task-irrelevant motion onset fails to capture attention. This interpretation implies that as perceptual resources are consumed, there is a decrease in the ability of a salient but task-irrelevant stimulus to capture attention. Note, however, that our lack of a significant flanker effect in the low-load, static-flanker condition is at odds with load theory. A strict account of load theory predicts load to be the sole determinant of distractor processing; therefore, both flanker types - those with motion and those without - should have produced flanker effects. Our lack of a flanker effect in the low-load, static-flanker condition seems to suggest that perceptual load interacts with bottom-up attentional capture parameters (e.g., motion onset). We should hasten to add that load theory was developed with data from displays that did not manipulate salient, bottom-up factors such as motion onset. Our results do not represent a fatal blow to load theory but, instead, point to previously unexplored conditions that would require some revision to load theory.

The lack of a flanker effect in the low-load, static-flanker condition also appears to run counter to previous results in 
the literature. Eltiti, Wallace, and Fox (2005) investigated attentional capture with a task very similar to that both in the present experiments and in our previous work (Cosman \& Vecera, 2009). In their most relevant experiment, Eltiti et al. found that when participants reported the identity of an offset target in a low-load display, an offset flanker produced a reduced $(5-\mathrm{msec})$ but significant flanker effect (see Experiment 3 in Eltiti et al., 2005). This effect was discussed in terms of contingent attentional control (Folk et al., 1992): The observers were set for an offset target, and offset flankers that matched this attentional control setting produced a flanker effect. Onset flankers that did not match the attentional control setting did not produce a flanker effect. In the present experiments, our targets and flankers appeared as offsets, yet we failed to find a flanker effect in the low-load, static-flanker condition.

However, along with the apparent differences between our results and those reported by Eltiti et al. (2005), there are also a number of important methodological differences between the studies. Most important, in our displays, there were two flanker letters present on each trial-one of which was presented as an offset and the other an abrupt onset (Cosman \& Vecera, 2009) or motion onset (the present experiments). In Eltiti et al.'s procedure, only one flanker appeared. In our procedure, each display contained a neutral flanker and a compatible or incompatible flanker. In the low-load, static-flanker condition of the present experiments, spatial attention was likely captured by and directed to the location of the motion onset flanker. Such a shift would presumably direct attention away from the congruent or incongruent flanker and toward the neutral flanker, and as a result, the neutral flanker would receive increased processing relative to the static congruent or incongruent flanker at the now unattended location. Because attention was directed to the neutral motion onset flanker, responses would have been unaffected and no flanker effect would have resulted — exactly the pattern of results that we report. This interpretation is in line with studies showing that distractor processing in low-load displays is modulated when endogenous attention is focused on a particular location in advance of the flanker letter (Johnson, McGrath, \& McNeil, 2002) and provides evidence that the exogenous capture of attention away from distracting task-irrelevant information may similarly allow observers to effectively ignore such information in low-load displays.

Finally, note a possible limitation with our design. Specifically, flanker effects were computed using incongruent minus congruent rather than incongruent minus neutral RTs, because a neutral flanker was present on every trial, making incongruent minus neutral calculations of flanker interference impossible. It has been argued that because of their feature overlap with the target, congruent flankers may exert effects at an early level and may produce changes in RTs to the target letter outside of attentional selection processes (Lavie, 1995; but see Lavie et al., 2004, for evidence contrary to this). However, given that we have recently replicated the effects of Cosman and Vecera (2009) using both incongruent-congruent and incongruent-neutral comparisons (Cosman \& Vecera, in press), along with the fact that we were interested in using flanker interference as an assay for capture (and observed capture by motion onset but not by static flanker letters under low-load conditions), we can be fairly certain that perceptual load exerted an effect on capture by motion onsets and that the effect was not an artifact of our experimental design.

\section{Relationship With Previous Studies of Motion Capture}

The results of the present study are, to our knowledge, the first to show that attention capture by the onset of new motion can be attenuated under conditions of high perceptual load. This contrasts with a recent demonstration that attentional control settings for color are not sufficient for overriding capture by new motion (Al-Aidroos, Guo, \& Pratt, 2010). Specifically, Al-Aidroos et al. used a contingent attentional capture paradigm to examine the influence of attentional control settings on capture by the onset of rotational motion. In their task, observers were presented with white boxes to the left or right of fixation, and each box had a white object inside of it. The observers were instructed to monitor each object for a color change (from white to red) and to respond to the location (left or right of fixation) of the target object undergoing the color change, which induced an attentional set for color. Directly before this color change, one of the target locations was cued, by undergoing either a color change (from white to red) or a rotational motion for $100 \mathrm{msec}$. The results showed that even though the participants' attentional control settings were for color, motion onsets captured attention and produced a cuing effect. This finding is in opposition to those of previous studies of contingent orienting (e.g., Folk et al., 1992), leading Al-Aidroos et al. to conclude that the onset of motion may be unique in its ability to capture attention in a purely stimulus-driven manner.

Our results seem to conflict with Al-Aidroos et al.'s (2010) findings, because we have demonstrated that capture by motion onsets is not mandatory but is modulated by perceptual load. We would argue that Al-Aidroos et al.'s two-item displays were low in perceptual load, which may have allowed the motion onset to override the attentional control settings for color. It may be possible that attentional control settings interact with perceptual load and would guide attention more strongly and allow attention to resist stimulus-driven capture by motion onsets if highload displays were used in a contingent capture paradigm similar to that of Al-Aidroos et al. The dissociation between our results and those of Al-Aidroos et al. suggest a difference in the mechanisms responsible for producing load-dependent and control-settings-dependent attenuation of capture. As was discussed in the previous section, it is possible that bottom-up and top-down factors interact to produce load effects, and this may cause load effects to have a stronger ability to attenuate capture than top-down factors alone.

The present results also appear inconsistent with those of accounts of capture by motion onsets that posit a unique status for these stimuli on the basis of their behavioral relevance (Abrams \& Christ, 2003, 2005, 2006; Franconeri 
\& Simons, 2003, 2005). More specifically, Franconeri and Simons (2003) demonstrated that looming motion - as opposed to receding motion - is more likely to capture attention in a stimulus-driven manner, presumably because it signals the need for imminent action on the part of the observer (however, see Abrams \& Christ, 2005, for evidence of capture by receding motion). Given that we found no evidence of capture by looming motion in Experiment 3, it seems that behavioral urgency might be trumped by display complexity and search difficulty. Again, however, we should acknowledge that the behavioral urgency account was developed from paradigms in which perceptual load was not manipulated per se, but in which set size was manipulated more generally in order to examine effects of capture on search rate. Given that set size manipulations are not necessarily synonymous with load, this is an important distinction, and one that is often ignored in the literature examining perceptual load effects. For example, we have unpublished data showing that increases in set size are themselves not sufficient to drive load effects in a traditional high-load search task (e.g., Lavie, 1995) but, instead, that the limited display duration used in this task is a major determinant in whether load effects emerge (Roper, Cosman, \& Vecera, 2010). With this in mind, our present results provide a unique demonstration that behavioral relevance might have limits and might not operate effectively in complex, high-load displays.

As a final point, note a major difference between the present study and previous studies demonstrating stimulusdriven attentional capture by motion onsets (e.g., Abrams \& Christ, 2003, 2005; Franconeri \& Simons, 2003, 2005), especially because in the previous studies motion onset capture was found in displays with large set sizes (as in our high-load displays). In our capture paradigm, the motion onset is always completely task irrelevant and occurs in a task-irrelevant location. In previous studies on motion capture, a variant of the irrelevant-feature search paradigm has typically been employed, in which target and distractor items were equally likely to appear as a motion onset. In this way, the motion onset is considered an irrelevant feature of the search display, because it is not predictive of the target location on a given trial. However, the fact that the target is defined by a salient motion onset on some trials potentially makes the motion onset task relevant. Furthermore, the task-irrelevant motion always occurs at a location that could theoretically be occupied by a target (i.e., within the search display vs. outside of it). These factors could lead observers to either implicitly or explicitly set their attention system to be captured by motion onsets. Our present results are consistent with this analysis and argue that task relevance, in addition to load, may be a major factor in whether a given stimulus will capture attention.

\section{Conclusion}

Our findings are in line with previous evidence showing that tasks high in perceptual load (Cosman \& Vecera, 2009; Santangelo et al., 2008; Santangelo \& Spence, 2007) or cognitive load (Boot, Brockmole, \& Simons, 2005) can modulate attentional capture by abrupt onsets. The present results extend these previous findings into the realm of motion onsets and suggest that perceptual load influences capture by dynamic stimuli more generally. It is possible that during attentionally demanding search tasks, irrelevant distractor items can be suppressed to allow attention to be more effectively focused only on task-relevant items, even when these items are defined by a motion onset or other salient, dynamic attributes. Therefore, a primary determinant of whether attention will be captured in a stimulus-driven manner may be the perceptual load of the primary task being performed.

\section{AUTHOR NOTE}

This research was supported in part by Grant BCS 03-39171 from the National Science Foundation awarded to the second author. We thank James Brockmole, Andrew Leber, Brad Gibson, and an anonymous reviewer for helpful comments on an earlier draft of the manuscript. Correspondence concerning this article should be addressed to J. D. Cosman, Departments of Neuroscience and Psychology, E305 Seashore Hall, University of Iowa, Iowa City, IA 52242-1407 (e-mail: joshua -cosman@uiowa.edu).

\section{REFERENCES}

Abrams, R. A., \& Christ, S. E. (2003). Motion onset captures attention. Psychological Science, 14, 427-432.

Abrams, R. A., \& Christ, S. E. (2005). The onset of receding motion captures attention: Comment on Franconeri and Simons (2003). Perception \& Psychophysics, 67, 219-223.

Abrams, R. A., \& Christ, S. E. (2006). Motion onset captures attention: A rejoinder to Franconeri and Simons (2005). Perception \& Psychophysics, 68, 114-117.

Al-Aidroos, N., Guo, R. M., \& Pratt, J. (2010). You can’t stop new motion: Attentional capture despite a control set for colour. Visual Cognition, 18, 859-880.

Biggs, A. T., \& Gibson, B. S. (2010). Competition between color salience and perceptual load during visual selection can be biased by top-down set. Attention, Perception, \& Psychophysics, 72, 53-64.

Boot, W. R., Brockmole, J. R., \& Simons, D. J. (2005). Attention capture is modulated in dual-task situations. Psychonomic Bulletin \& Review, 12, 662-668.

Brainard, D. H. (1997). The Psychophysics Toolbox. Spatial Vision, 10, 433-436.

Cosman, J. D., \& Vecera, S. P. (2009). Perceptual load modulates attentional capture by abrupt onsets. Psychonomic Bulletin \& Review, 16, 404-410.

Cosman, J. D., \& Vecera, S. P. (in press). Attentional capture under high perceptual load. Psychonomic Bulletin \& Review.

Couperus, J. W. (2009). Implicit learning modulates selective attention at sensory levels of perceptual processing. Attention, Perception, \& Psychophysics, 71, 342-351.

Cousineau, D. (2005). Confidence intervals in within-subjects designs: A simpler solution to Loftus and Masson's method. Tutorials in Quantitative Methods for Psychology, 1, 42-45.

Eltiti, S., Wallace, D., \& Fox, E. (2005). Selective target processing: Perceptual load or distractor salience? Perception \& Psychophysics, 67, 876-885.

Folk, C. L., Remington, R. W., \& Johnston, J. C. (1992). Involuntary covert orienting is contingent on attentional control settings. Journal of Experimental Psychology: Human Perception \& Performance, 18, 1030-1044.

Franconeri, S. L., \& Simons, D. J. (2003). Moving and looming stimuli capture attention. Perception \& Psychophysics, 65, 999-1010.

Franconeri, S. L., \& Simons, D. J. (2005). What dynamic signals capture attention: A reply to Abrams and Christ (2005). Perception \& Psychophysics, 67, 962-966.

Gibson, B. S., \& Bryant, T. A. (2008). The identity intrusion effect: Attentional capture or perceptual load? Visual Cognition, 16, 182199.

Johnson, D. N., McGrath, A., \& McNeil, C. (2002). Cuing interacts 
with perceptual load in visual search. Psychological Science, 13, 284287.

JONIDES, J., \& YANTIS, S. (1988). Uniqueness of abrupt visual onset in capturing attention. Perception \& Psychophysics, 43, 346-354.

LAVIE, N. (1995). Perceptual load as a necessary condition for selective attention. Journal of Experimental Psychology: Human Perception \& Performance, 21, 451-468.

LaVIE, N., \& Cox, S. (1997). On the efficiency of attentional selection: Efficient visual search results in inefficient rejection of distraction. Psychological Science, 8, 395-398.

Lavie, N., \& DE Fockert, J. W. (2003). Contrasting effects of sensory limits and capacity limits in visual selective attention. Perception \& Psychophysics, 65, 202-212.

Lavie, N., Hirst, A., De Fockert, J. W., \& Viding, E. (2004). Load theory of selective attention and cognitive control. Journal of Experimental Psychology: General, 133, 339-354.

Leber, A. B., \& Egeth, H. E. (2006). It's under control: Top-down search strategies can override attentional capture. Psychonomic Bulletin \& Review, 13, 132-138.

Loftus, G. R., \& Masson, M. E. J. (1994). Using confidence intervals in within-subject designs. Psychonomic Bulletin \& Review, 1, 476490.

Martin-Emerson, R., \& Kramer, A. F. (1997). Offset transients modulate attentional capture by sudden onsets. Perception \& Psychophysics, 59, 739-751.
Norman, D. A., \& Bobrow, D. G. (1975). On data-limited and resourcelimited processes. Cognitive Psychology, 7, 44-64.

Roper, Z., Cosman, J. D., \& Vecera, S. P. (2010). Presentation duration as a major determinant of perceptual load during search. Unpublished manuscript.

Santangelo, V., Finoia, P., Raffone, A., Belardinelli, M. O., \& SPENCE, C. (2008). Perceptual load affects exogenous spatial orienting while working memory load does not. Experimental Brain Research, 134, 371-382.

Santangelo, V., \& Spence, C. (2007). Multisensory cues capture spatial attention regardless of perceptual load. Journal of Experimental Psychology: Human Perception \& Performance, 33, 1311-1321.

TheEuwes, J. (1994). Stimulus-driven capture and attentional set: Selective search for color and visual abrupt onsets. Journal of Experimental Psychology: Human Perception \& Performance, 20, 799-806.

Theeuwes, J., Kramer, A. F., \& Belopolsky, A. V. (2004). Attentional set interacts with perceptual load in visual search. Psychonomic Bulletin \& Review, 11, 697-702.

Torralbo, A., \& Beck, D. M. (2008). Perceptual load-induced selection as a result of local competitive interactions in visual cortex. Psychological Science, 19, 1045-1050.

(Manuscript received May 22, 2009;

revision accepted for publication June 25, 2010.) 